\title{
Meet the PAREO Functions: Towards Reliable and Available Wireless Networks
}

\author{
Remous-Aris Koutsiamanis ${ }^{\dagger}$, Georgios Z. Papadopoulos ${ }^{\dagger}$, Tomas Lagos Jenschke ${ }^{\dagger}$, \\ Pascal Thubert ${ }^{\ddagger}$ and Nicolas Montavont ${ }^{\dagger}$ \\ $\dagger$ IMT Atlantique, Irisa, France, Email: \{firstname.lastname\}@imt-atlantique.fr \\ ¥ Cisco Systems, France, Email: pthubert@cisco.com
}

\begin{abstract}
Deterministic networking allows carrying data flows with low data-loss rates and with bounded latency. A typical usecase is the convergence of Operational Technology (OT) with Information Technology (IT), also known as the Industrial Internet. Wireless networks operate on a shared communication medium where the potential external interference along with multi-path fading impact data packet delivery. By employing diversity in the time, frequency and spatial domains, wireless technologies with scheduled transmissions, such as IEEE Std 802.15.4-2015 Time Slot Channel Hopping (TSCH), can mitigate those effects and provide Reliable and Available Wireless (RAW) communications that approach determinism. Nevertheless, a radio link operating in the ISM band still needs to handle collisions and possibly re-transmission. Therefore, It takes redundant links and paths to provide both the high availability and the near consistent reliability that industrial applications require. In this paper, we present the Packet Automatic Repeat reQuest (ARQ), Replication and Elimination (RE), and Overhearing (PAREO) functions to further increase the Quality of Service $(\mathrm{QoS})$ in industrial networks, even when implemented on top of best-effort traffic in a shared network. The results show that PAREO provides up to approximately 6 times lower Packet Error Rate (PER) and up to approximately $10 \%$ higher energy consumption than the default RPL implementation with 7 retransmissions, while keeping jitter and latency as low as default RPL with 1 retransmission.
\end{abstract}

Index Terms-IoT, LLN, RPL, IEEE802.15.4-TSCH, Multipath, ARQ, PAREO, RAW

\section{INTRODUCTION}

Industry 4.0 aims at high-level automation of production chains through systems that can provide security, adaptivity and ease of control. If we take into account the evolution of Internet of Things (IoT) technologies, many of these requirements in the industry are close to being met. Some characteristics of the IoT technology are its adaptivity to changes, its portability, its low power consumption and its variety of wireless technologies in which it can work.

The micro-controllers used in these sensors are certainly limited but are powerful enough to enable a large array of precise monitoring and control applications. Despite often being battery-powered, their design usually supports multi-year operation. The wireless interfaces support short, medium or long range communication with relatively good reliability, though not as high as wired solutions can provide. Additionally, due to the constrained nature of the devices, either due to programming errors or due to a temporary loss of access to the wireless medium, devices can become temporarily inaccessible.

Despite these shortcomings, Wireless Sensor Networks (WSNs) are posed to play a significant role since a large part of the drive towards automation in Industry 4.0 is being able to monitor and control as many parts of the production chain as possible while being able to perform changes without prohibitive cost. By virtue of operating wirelessly and without a need for a wired power source, it becomes easy to reconfigure the sensors to adapt to new needs of equipment configurations.

The problem lies in merging the wireless environment with the needs of the production chains, given that industry is based on quality-of-service solutions, while wireless technologies generally offer best-effort solutions [1], [2]. In this sense, the biggest issue to be addressed is the reliability and predictability that a network must offer for production chains. While there have been standardization efforts to provide deterministic networking, such as at the Deterministic Networking (DetNet) Working Group (WG) in the Internet Engineering Task Force (IETF), until recently, most efforts were focused on wired networks [3].

The existing compensation for this is to perform a certain number of data re-transmissions, which impacts jitter and, thus, makes the network less predictable. Additionally, re-transmissions do not solve the problem of temporarily inaccessible forwarding nodes. To address these issues, we work on top of the IEEE Std 802.15.42015 TSCH MAC protocol [4] because it offers the functionality to approach deterministic networking on a wireless medium.

In this direction, a new line of work is being considered at the IETF with a focus on wireless, called Reliable and Available Wireless (RAW) [5]. RAW is a layer-3 approach to determinism that will operate over a wide range of scheduled radios [6], including but not limited to TSCH, 5G and Wi-Fi 6. RAW considers the forwarding operation along a complex path, called a Track, as mostly a Destination-Oriented Directed Acyclic Graph (DODAG) towards a destination, with possibly parallel links and paths for availability, and possibly heterogeneous technologies along the path. A Track exhibits multiple possibilities of redundancy that RAW leverages to counterbalance the inherent fuzziness of a single-hop wireless link, some of which, like overhearing, are specific to radios.

The contributions of this paper are the application of the RAW concept of Packet Automatic Repeat reQuest (ARQ), Replication and Elimination (RE), and Overhearing (PAREO) to provide an acceptable trade-off of energy and bandwidth versus reliability and availability over a 6TiSCH Track [7]. We implement PAREO within the Contiki OS and we use COOJA simulator to perform an extensive evaluation. Validating our approach, the results show that we achieve higher reliability, lower latency and jitter, at a small energy consumption cost. 


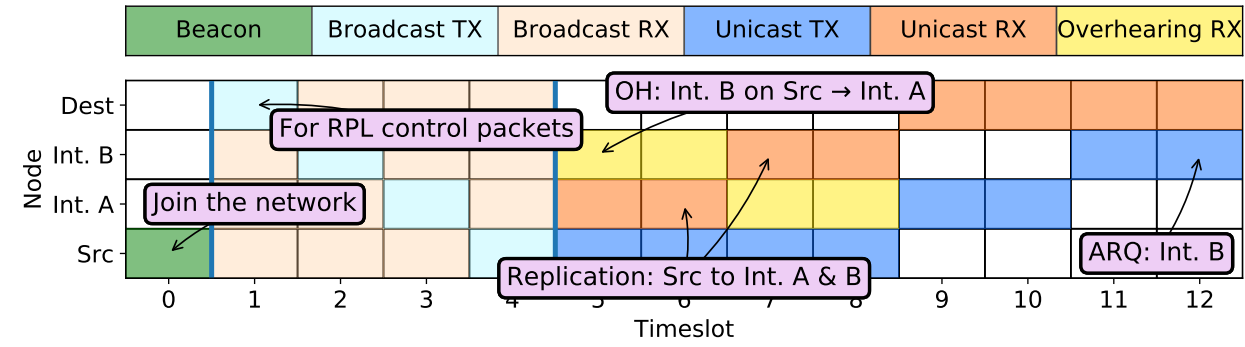

Fig. 1. TSCH example schedule showing Replication, Overhearing, and ARQ operations.

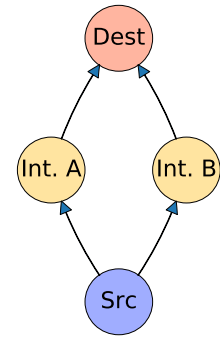

Fig. 2. TSCH example network topology.

\section{TECHNICAL BACKGROUND}

\section{A. Terminology}

High expectations are placed on the word deterministic, and such expectations might be beyond what can be achieved on wireless links, in particular in the ISM band that is shared with uncontrolled interferers. It seems safer to avoid associating deterministic and wireless, and the RAW work preferred to introduce its own terminology.

PAREO functions inherit and extend from the DetNet concept of Packet Replication Elimination and Ordering Functions (PREOF) [8]. This document also reuses the concept of Track as defined by 6TiSCH [7] to represent a complex path towards a destination, typically a DODAG.

By reliability, we express a measure of the probability that an item will perform its intended function for a specified interval under stated conditions. For this paper and the work at RAW in general, the service that is expected is delivery within a bounded latency and a failure is when the packet is either lost or delivered too late. RAW expresses reliability in terms of Mean Time Between Failure (MTBF) and Maximum Consecutive Failures (MCF).

On the other hand, with availability, we express the relative amount of time during which a path operates correctly, i.e. uptime/(uptime+downtime). Because a serial wireless path may not be good enough to provide the required availability, and even 2 parallel paths may not be enough over a longer period of time, the RAW availability implies a path that is a lot more complex than what DetNet typically envisages, in other words, a Track.

\section{B. IEEE 802.15.4-TSCH}

IEEE 802.15.4-Time Slot Channel Hopping (TSCH) defines a Medium Access Control (MAC) extension that enables scheduled transmission with pseudo-random channel-hopping. This technique reduces the chances of collision and improves the energy efficiency with the capability to stay in deep sleep between scheduled transmissions. To achieve this, TSCH uses a time-slotted medium access method, where communication between nodes is performed within precise time-synchronized windows called time-slots. This allows for a more reliable access to the wireless medium, which is shared and probabilistic by nature. In addition, IEEE $802.15 .4-\mathrm{TSCH}$ incorporates the technique of channel hopping to avoid multi-path fading and potential external interference in wireless environments and to support parallel transmission in different channels.

As the combination of these two techniques, in IEEE 802.15.4-TSCH the network resource unit is a pair of time-slot and channel, called a cell. For each cell a number of configuration options can be set, including the source and destination addresses of the communicating nodes, whether the cell is reserved for broadcast or unicast, if an acknowledgment is required from the receiver, and others. Multiple cells are organized in a slotframe, which has a specified duration, and which repeats regularly for the lifetime of the network.

These features allow fine control and predictability over how and when nodes communicate in the network, with the main requirement being that nodes can remain time-synchronized within strict bounds to be able to perform the same steps in the slotframe at the same time.

\section{IPv6 Routing Protocol for Low-Power and Lossy Networks $(R P L)$}

RPL [9] is a proactive distance-vector routing protocol produced by the Routing Over Low power and Lossy networks (ROLL) IETF WG. RPL proactively organizes network nodes into a special case of a Directed Acyclic Graph (DAG) called a DODAG. In each DODAG there is a single node considered to be the "root" (or destination) which configures significant network parameters and also serves as the gateway to other non-RPL networks. In this topology, upstream traffic is the network traffic from non-root nodes towards the root. Downstream traffic flows in the opposite direction.

In order to form the topology, each non-root node has the notion of a Parent Set (PS) of nodes, nodes which it may use to forward traffic upstream. In the same sense, each node may have children nodes, for which it may forward upstream traffic. If a node has no children, then it is a leaf node. Additionally, each non-root node selects one node among its PS to act as a Preferred Parent (PP) node. This PP will be used to forward packets upstream.

The process of creating the topology starts from the root and proceeds "downwards" towards the leaf nodes, iteratively connecting nodes as they are detected. When a node has the option to connect via more than one parent nodes, it selects its PP to be the one which minimizes a configurable distance metric. The value of this distance metric is called its "rank" in RPL terminology. The way in which RPL calculates the 
rank of each node is given by the Objective Function (OF), an abstraction of this decision mechanism in RPL. In this work we use the default metric, Expected Transmission Count (ETX) [10].

Finally, RPL has three types of control packets for its operation: The DODAG Information Object (DIO), the Destination Advertisement Object (DAO), and the DODAG Informational Solicitation (DIS), with the DIO being the only one required to allow upstream traffic and to form the topology. The DAO is used for downstream route generation and the DIS for optimized network joining.

\section{PAREO FUNCTIONS}

The PAREO methodology consists of a set of functions with the aim to improve network reliability and predictability and energy efficiency in Low-power and Lossy Networks (LLNs). The individual functions, illustrated in Fig. 1 and 2, complement each other towards this goal but work somewhat independently of each other. The only point where they need to collaborate directly is in the definition of the TSCH schedule, where depending on which functions are used, the schedule needs to be modified accordingly. In the following sections we present each constituent PAREO function and how they interact.

\section{A. Automatic Repeat reQuest (ARQ)}

The ARQ function performs the re-transmission of data packets when a previous transmission failed [11]. In our context, we employ a link layer ARQ so the decision for re-transmission is local to the transmitting node. This function requires the use of an acknowledgment (ACK) control packet for each data transmission. Fortunately, TSCH allows cells to be configured to require an ACK, which is transmitted from the receiver to the sender within the same cell as the data transmission.

Since in our context only one packet will be "in flight" at any one point in time in a given cell, we can use the Stop-and-Wait variation of $A R Q$, as described below. The function starts by sending a packet and setting a short timeout to await an ACK (within the same cell). If the ACK is received from the receiver, the transmission is marked as successful and finishes. If no ACK is received, then the transmission is marked as temporarily failed and is re-scheduled again in the future. For the same data packet, each time a (re-)transmission fails, a counter is incremented. If the counter reaches a threshold, named the Re-Transmission (RTX) count, then the packet is marked as permanently failed and it is not scheduled again.

The scheduling of the re-transmission is tightly coupled with the structure of the TSCH schedule. Depending on the schedule, the timing of re-transmission attempts can vary significantly: from immediately after a temporary failure to quite later during the next repetition of the slotframe. While it is possible to include almost any number of re-transmission attempts for each pair of nodes in one slotframe, the more reserved cells for potential re-transmissions, the largest the slotframe size.

\section{B. Replication and Elimination (RE)}

The RE function modifies packet forwarding to send a packet not only to the PP of a node but also to other nodes in the
PS [12]. In the default implementations of RPL, each node only uses one node to forward packets (i.e., the PP). The problem with that approach is that if the PP fails for any reason, all packets from its children will be discarded until a new parent is selected. However, selecting a new parent requires one of two time-consuming processes, either local or global repair, during which time connectivity is not available. If the queue fills up with connectivity still not restored, packets will start being dropped. With RE we do not avoid having to perform local or global repair to select a new PP, but having established two or more parents where a packet can be sent, we can maintain connectivity and avoid dropped packets due to an inaccessible parent node.

In order to make this possible, a packet is cloned and each copy is forwarded to a different parent node, called an Alternative Parent (AP), in the PS. Performing packet replication operation creates multiple copies of the same packet, which traverse independently through the network. In order to avoid a situation where each received packet copy results in a further independent set of packet copies being forwarded, resulting in a flood, a packet elimination function is implemented.

Packet elimination uses a unique 32-bit packet ID added in the header of each packet sent (stored in the IPv6 RPL Option extension header in our implementation) to identify packet copies. The unique ID of the last $k$ packets forwarded are recorded in a Least Recently Used (LRU) or Last In, First Out (LIFO) unique-id-history cache in each node, so when a new packet arrives for forwarding, the presence of its unique ID is checked in the unique-id-history cache. If it is found inside, the packet is dropped without forwarding since this means that a copy has been previously already forwarded. Otherwise, the unique ID is added to the cache, potentially evicting another entry. The size $k$ of the unique-id-history cache can be set based on the maximum data traffic possible in the maximum end-to-end delay.

The AP selection is not trivial, and we previously proposed a methodology called Common Ancestor (CA) [13], [14]. More specifically, when a node $n$ is choosing an AP node, it ensures that the selected AP has in its PS the node which is the PP of $n$. In order to support this selection mechanism, additional information is carried in the DIO control message that all nodes broadcast. The information carried is the list of IPv6 addresses corresponding to the $m$ lowest ranking (and therefore most preferable) parents in node $n$ 's parent set. We have extended the operating system used (Contiki OS) to include this information in the DIO message, in the DAG Metric Container (MC) field. ${ }^{1}$

The interaction of RE with the TSCH schedule only concerns the required existence of cell(s) for transmitting packets from a node not only to its PP but also to the AP.

\section{Overhearing $(\mathrm{OH})$}

The overhearing function [15] can be used to take advantage of the shared nature of the wireless medium in order to increase reliability. This function allows a node to simultaneously forward a packet to multiple parent nodes, which by virtue of being in the PS are assumed to be within radio range. Overhearing

\footnotetext{
${ }^{1}$ https://github.com/ariskou/contiki/tree/draft-ietf-roll-nsa-extension
} 


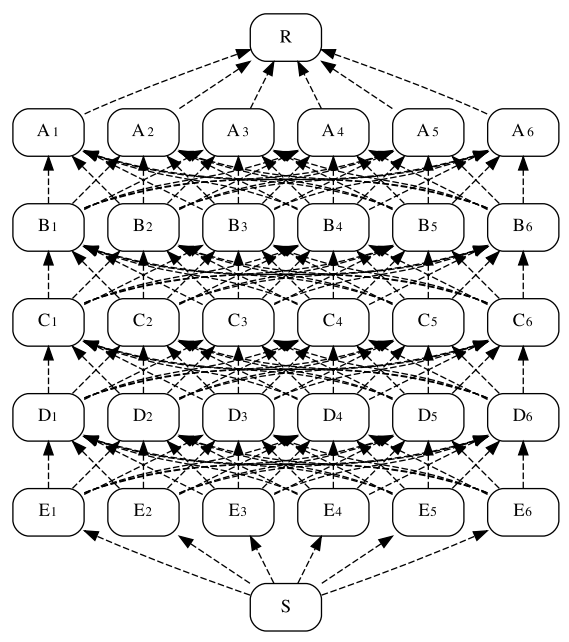

Fig. 3. Network topology used in the experimental evaluation.

only makes sense when RE is also used, working in addition to it to increase reliability without sacrificing transmission latency.

Since with this function the sender effectively broadcasts the packet and receiving nodes disable their MAC filter, two issues need to be addressed. Firstly, promiscuous overhearing is performed for data packets which are typically forwarded with ACK-ed unicast transmissions. However, multiple parents may receive the broadcast and attempt to respond with an ACK, potentially resulting in a collision if multiple nodes attempt it at the same time. This would lead the sending node to consider the transmission to have failed. Thus, it is important that only one node, i.e. the recipient indicated in the destination address field, responds with an ACK, while all other nodes silently receive the packet without sending an ACK. This capability is neither present by default in TSCH nor implemented in Contiki OS. We provided a solution by introducing $\mathrm{OH}$ cells, which accept packets sent to any destination (by disabling MAC filtering) but which suppress responding with an ACK even when one is requested.

Secondly, it is desirable to control the replication of packets via overhearing so that only the AP node forwards the packet and not any node that happens to overhear it. This is not supported in TSCH or RPL by default, thus we have evaluated two options to implement it. One way to do this is via the TSCH schedule, configuring only the AP to overhear when the node transmits. Another is by storing the address of the AP in the packet so even if other nodes receive it, they can check the stored AP address against their own and avoid forwarding. In our implementation we have opted for the latter since it simplifies the TSCH schedule management.

The interaction of overhearing with the TSCH schedule only concerns the required existence of receiver cell(s) for overhearing packets from a node to its AP.

\section{Performance EVAluation}

\section{A. Simulation Setup}

We performed extensive experiments to evaluate the trade-offs presented by the use of the RE, ARQ, and $\mathrm{OH}$ functions. More specifically, we evaluated with a network topology (shown in
Fig. 3) with 32 nodes, out of which, one source node (S), one destination/root node $(\mathrm{R})$ and 30 intermediate nodes $\left(A_{1} \ldots E_{6}\right)$.

The intermediate nodes form 5 layers $(A \ldots E)$ of 6 nodes (1..6) and every node in the network, except for the root, is within the radio communication range of all the nodes in the layers immediately above and below it. We evaluated a total of 32 scenarios which include the standard Single-Path (SP) forwarding mechanism, with different values for the number of MAC layer Re-Transmissions (RTX): 0, 1, 3, and 7 (for a total of 1, 2, 4, and 8 transmissions correspondingly). Additionally, we evaluate combinations of the PAREO operations: RE (no ARQ, no $\mathrm{OH}$ ), $\mathrm{RE}+\mathrm{OH}$ (no ARQ), RE + ARQ (no OH), and RE + OH + ARQ (i.e., PAREO), to evaluate the contribution of each operation individually. The MAC layer implementation used is TSCH with a single channel and the schedule used is pre-determined to allow one TX opportunity for each child-parent uplink when $R T X=0$ and two when $R T X \geq 1$. By taking into account the trade-off between the slotframe size and the end-to-end latency in TSCH networks, we therefore we have chosen to limit the number of transmission opportunities in a slotframe to 2 per child-parent link.

For each routing algorithm choice ( 8 in total, 4 single-path plus 4 multi-path) we set different link qualities (60\% and $75 \%)$ between all the nodes, while we also measure the impact of preferred parent killing. With preferred parent killing enabled, once the network is connected, we evaluate the ability of the routing algorithm to adapt to the failure of an important node in the topology. This process is normally time-consuming since even in the best case (local repair) a new PP needs to be picked and the root node to be informed of the new route. In the worst case (global repair), the whole structure of the network might need to be changed, with routing changes being required in multiple network nodes.

To achieve this, every 5 minutes we identify which node is on the preferred parent path from the source $S$ to the destination $\mathrm{R}$ among the nodes in the middle of the network ( $\mathrm{C}$ layer, nodes $C_{1} \ldots C_{6}$ ). Then, we completely disconnect this node from all its neighbors for the next 5 minutes while continuing to send data packets from the source node $S$ to the destination node R. After the 5 minutes pass, the node is reconnected to its neighbors, the new node on the preferred path on the $\mathrm{C}$ layer is identified and fully disconnected. This process stresses the ability of the routing algorithm to adapt to changes by specifically targeting nodes on the forwarding path from the source to the destination. For each scenario we executed 50 simulations with different random seed numbers, and in each simulation 100 packets were sent from the source node $\mathrm{S}$ to the destination node $\mathrm{R}$. Therefore, for each of the 32 scenarios, 5000 packet transmissions are made for a total of 160,000 for the whole work. We used Contiki OS, with modifications implementing the $\mathrm{RE}$ and $\mathrm{OH}$ operations, and the simulated network environment provided by COOJA.

\section{B. Simulation Results}

1) End-to-end (multi-hop) PDR: The results for reliability shown in Fig. 4 support the usefulness of PAREO. Single-path requires the SP RTX7 algorithm, with 7 re-transmissions and 8 total transmissions, in order to compete with PAREO. It can only 

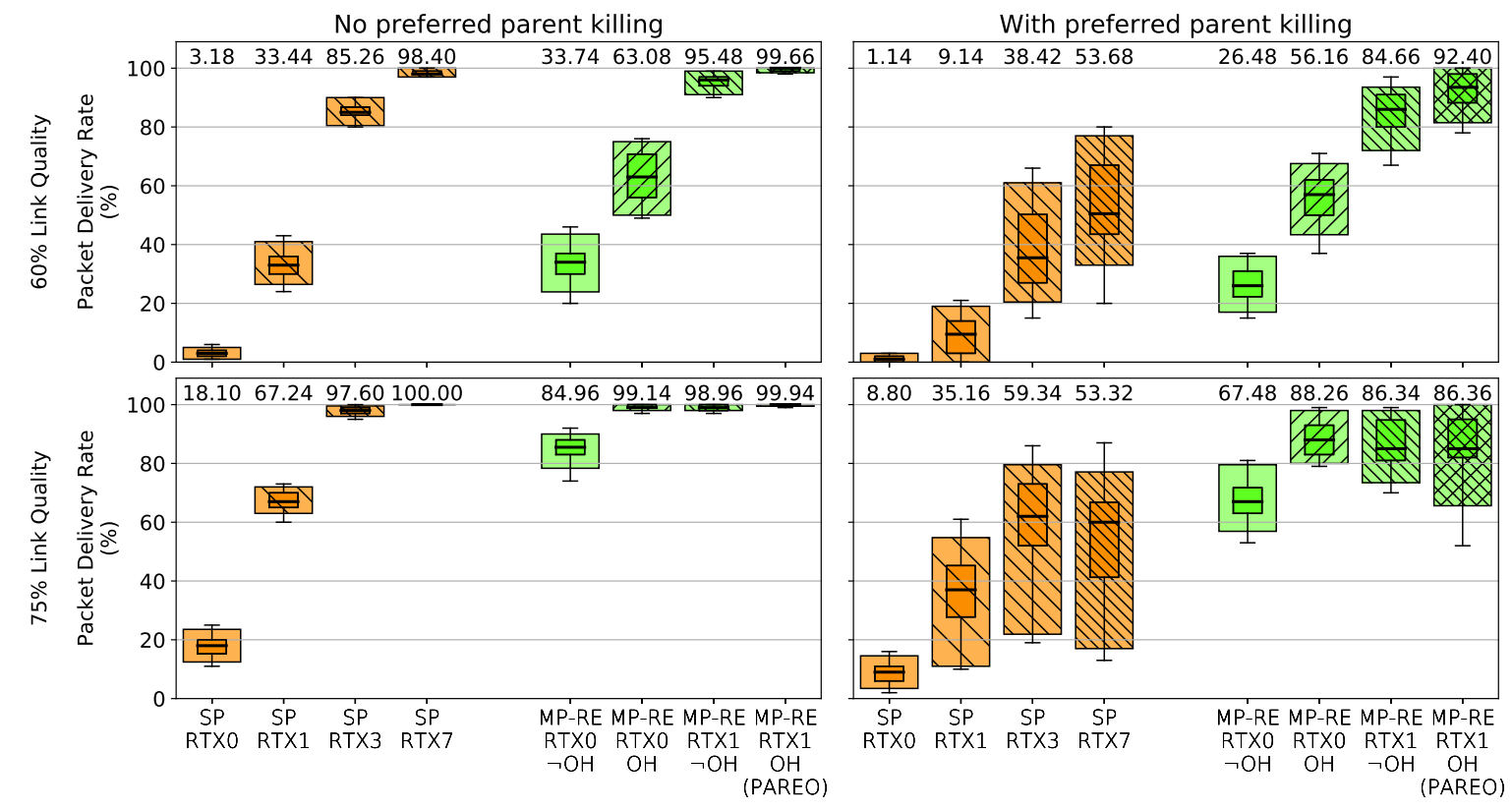

Fig. 4. End-to-end (multi-hop) Packet Delivery Rate (PDR) for data packets sent from node $\mathrm{S}$ to node R. The four quadrants express the four combinations of the preferred parent killing (with and without) and the link quality (60\% and $75 \%$ ) parameters. In each plot, the orange boxes correspond to the default Single-path RPL routing algorithm, while the green to our Multi-path with RE routing algorithm. Furthermore, the outer (lighter) box corresponds to the 5\%-95\% percentiles, the inner (darker) to the $25 \%-75 \%$ percentiles, the middle line to the median and the whiskers represent the minimum and maximum. On top of each box, the mean value is presented. Finally, in the labels, $\mathrm{OH}$ and $\neg \mathrm{OH}$ stand for with and without the overhearing feature, and RTX $n$ expresses the number of re-transmissions performed.

compete when the link quality is higher $(75 \%)$ and no preferred parent is killed (bottom right in Fig. 4). With either lower link quality $(60 \%)$ or preferred parent killing enabled, PAREO is superior in all cases to SP RTX7. In the case of $60 \%$ links with no preferred parent killing, PAREO has an approximately 5 times lower Packet Error Rate (PER), where PER $=100 \%-P D R$ :

$$
\begin{aligned}
P D R_{\mathrm{PAREO}}=99.66 \% \rightarrow & P E R_{\mathrm{PAREO}}=0.34 \% \\
P D R_{\mathrm{SPRTX} 7}=98.4 \% \rightarrow & P E R_{\mathrm{SPRTX} 7}=1.6 \% \\
& \frac{P E R_{\mathrm{SPRTX} 7}}{P E R_{\mathrm{PAREO}}} \approx 4.7 \times
\end{aligned}
$$

Moreover, the advantage that PAREO provides is especially pronounced when the link quality is lower $(60 \%)$ and preferred parent killing is enabled. In this case, PAREO $(P D R=92.4 \%)$ is much better than even SP RTX7 (PDR $=53.68 \%)$, translating to an approximately 6 times lower Packet Error Rate (PER).

Additionally, it can be seen that for RE, the biggest advantage is provided by the addition of ARQ, i.e. an extra MAC layer re-transmission, while $\mathrm{OH}$ helps but not to the same degree. More specifically, with $60 \%$ link quality and no preferred parent killing, ARQ reduces PER by 14.6 times while $\mathrm{OH}$ by 1.8 times. The results for $60 \%$ link quality with preferred parent killing are similar and in both cases PAREO achieves very high PDR due to access to alternative routes.

2) End-to-end (multi-hop) Delay and Jitter: It is interesting to note that the main advantage of using PAREO is that it can achieve low delay and low jitter at the same time, as shown in Fig. 5. All the combinations of the PAREO functions provide approximately the same performance given the same schedule, but for single-path, once the number of MAC layer re-transmissions attempted surpasses the number of transmission opportunities in the same slotframe (i.e. single-path with RTX 3, and 7) both delay and especially jitter are significantly impacted. While it is possible to just increase the number of transmission opportunities in the schedule to reduce jitter, this would nevertheless impact delay.

3) Energy Consumption: Since COOJA only provides power-tracking in terms of time spent in the different radio states, we have extrapolated the radio energy consumption of the whole network in $\mathrm{mJ}$ by using the radio power consumption values of the Zolertia Z1 mote which uses the $\mathrm{CC} 2420$ radio transceiver module. More specifically, we used the given specification sheet values $\left(P_{T X}=52.2 \mathrm{~mW} @ 3 \mathrm{~V}, P_{R X}=56.4 \mathrm{~mW} @ 3 \mathrm{~V}\right.$, $\left.P_{\text {Idle }}=1.28 \mathrm{~mW} @ 3 \mathrm{~V}\right)$ and that when being interfered the consumption is the same as when transmitting $\left(P_{T X}=P_{I N T}\right)$. Since the RTX0 scenarios use a shorter slotframe than the rest of the RTX scenarios, we report the energy consumption per slotframe, to allow meaningful comparisons. As shown in Fig. 6, the single-path scenarios generally have comparable energy consumption to the RE/ARQ/OH combinations, with higher RTX values predictably leading to higher energy consumption. It should be noted that the energy consumption measured comprises the cost sending both data and control packets.

\section{Discussion}

The main objective of PAREO is to ensure high reliability and fault-tolerance in the presence of temporarily unavailable nodes while also minimizing latency and jitter. To achieve this goal, PAREO takes advantage of the physical properties of wireless technologies via packet replication and elimination as well 

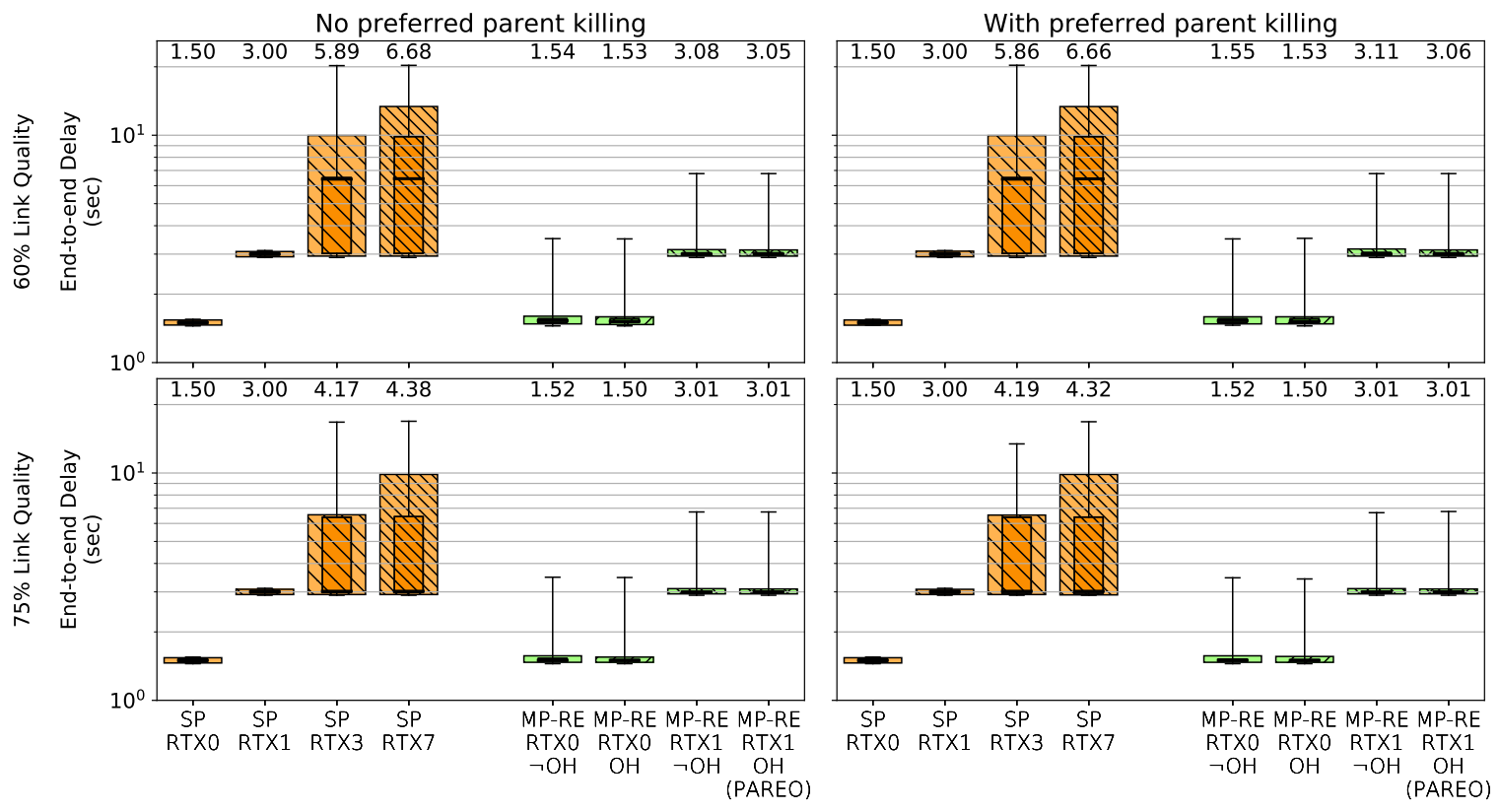

Fig. 5. End-to-end (multi-hop) Delay and Jitter (Percentiles of Delay) for data packets sent from node $\mathrm{S}$ to node R. The four quadrants express the four combinations of the preferred parent killing (with and without) and the link quality (60\% and $75 \%$ ) parameters. In each plot, the orange boxes correspond to the default Single-path RPL routing algorithm, while the green to our Multi-path with RE routing algorithm. Furthermore, the outer (lighter) box corresponds to the 5\%-95\% percentiles, the inner (darker) to the $25 \%-75 \%$ percentiles, the middle line to the median and the whiskers represent the minimum and maximum. On top of each box, the mean value is presented. Finally, in the labels, $\mathrm{OH}$ and $\neg \mathrm{OH}$ stand for with and without the overhearing feature, and RTXn expresses the number of re-transmissions performed.

as overhearing, thereby increasing the number of opportunities for a packet to reach its destination.

The result is a trade-off regarding energy efficiency, which is increased to achieve the previous aims. The main reason for this is the overhearing operation, and specifically due to our implementation which set all nodes in the PS of a node in the listening state. A more energy efficient but complicated solution would only set the AP node in the listening state.

\section{RELATED WORK}

Le et al. [16] propose and evaluate an Energy-awareness Load Balancing (ELB) protocol, a Fast Local Repair protocol (FLR), and their combination (ELB-FLR). The ELB protocol utilizes a pair of metrics, hop-counting and residual energy, and calculates node ranks from the node hop-count (appropriately scaled) minus the remaining energy in the node. Then an AP is selected for multi-path routing as the second lowest rank-providing parent. In the case of FLR, the focus is on the increase of path redundancy for high priority situations, by also using sibling nodes to forward packets. This work results in increased PDR and relatively low overhead, however, it provides no latency or jitter guarantees while also increasing energy consumption.

Minet et al. [17] examine different multi-path routing patterns: "Disjoint", "Triangle" and "Braided", with each providing an alternative path to increase reliability. Their results illustrate different performance trade-offs depending on the pattern used. Our multi-path routing is similar to the "Braided" pattern described. However, we develop our solution based on the standard RPL protocol, we perform our evaluation using a realistic Contiki OS environment and a more demanding network topology. Additionally, our solution solves the problem of flooding in the more complex topology by constraining the multi-path routing despite the availability of multiple potential alternative parents for each node.

Jin et al. [18] describe BOOST, an IEEE 802.15.4-TSCH-based scheduler and multi-path routing solution to increase reliability and energy efficiency. BOOST consists of a custom multi-path routing implementation and an autonomous scheduler which organizes nodes into layers, and which schedules communication based on each node's layer id. While this method is shown to provide high PDR and energy efficiency, no results are shown for latency and jitter. Additionally, the system is evaluated using a Matlab-based simulation, which might not take into account all the complicated issues in real IoT environment. Our PAREO methodology is also based on IEEE 802.15.4-TSCH, but it re-uses the standard RPL protocol with light modifications. Furthermore, we provide latency and jitter guarantees while performing our evaluation in a more realistic Contiki OS environment.

\section{CONCLUSION}

There is a great opportunity to achieve increased efficiency in the industrial field as part of an ongoing modernization wave. As part of that, the option of utilizing wireless networks to monitor and control industrial equipment brings enhanced flexibility and lower cost, by replacing wired installations. However, it is critical that wireless communications provide high quality-of-service guarantees in order to be able to replace wired communications in most cases.

These guarantees consist mainly of packet delivery rate, delay and jitter bounds on network traffic and fault-tolerance 

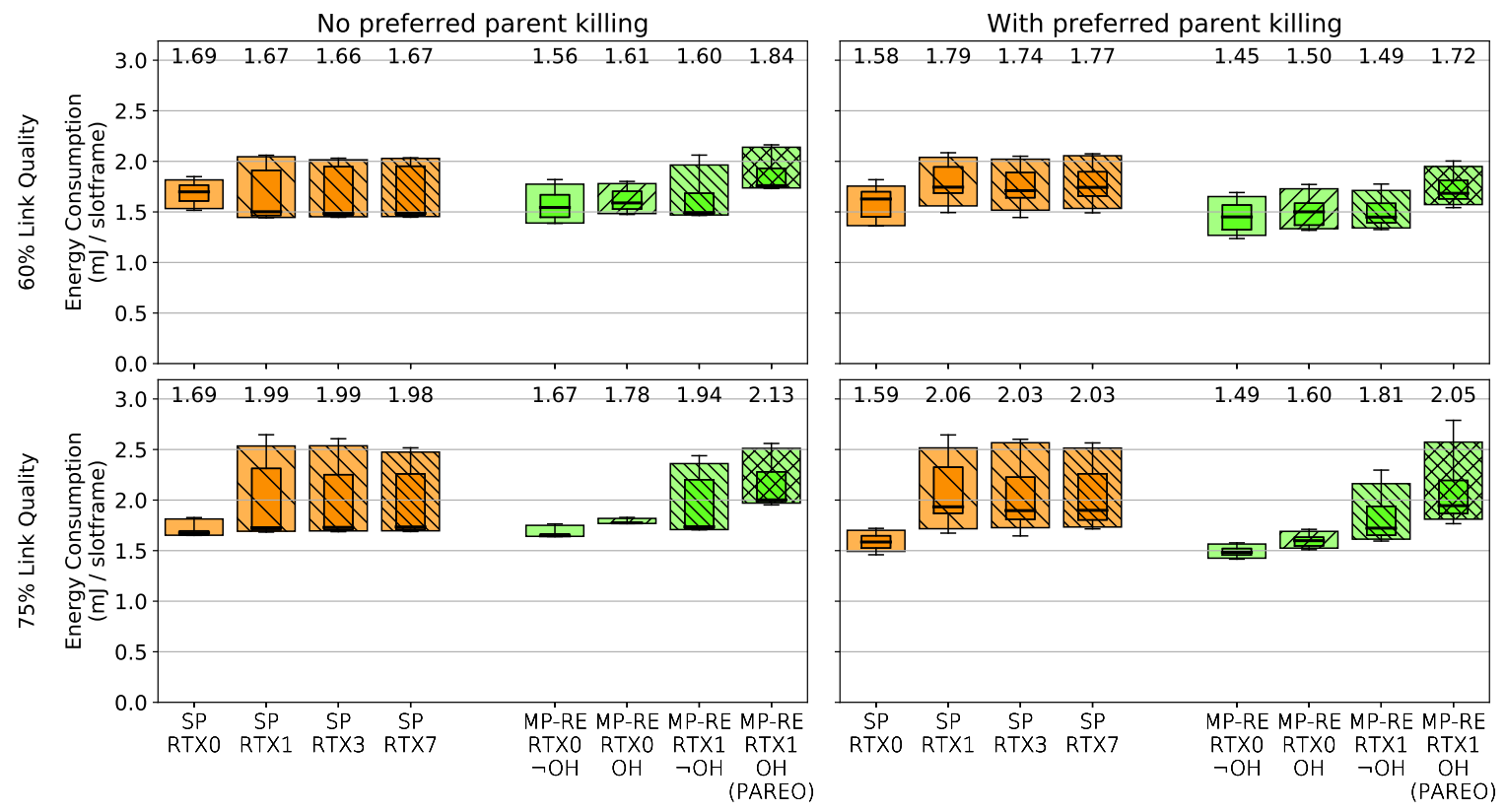

Fig. 6. Energy consumption per node per slotframe due to radio operation. The four quadrants express the four combinations of the preferred parent killing (with and without) and the link quality $(60 \%$ and $75 \%)$ parameters. In each plot, the orange boxes correspond to the default Single-path RPL routing algorithm, while the green to our Multi-path with RE routing algorithm. Furthermore, the outer (lighter) box corresponds to the 5\%-95\% percentiles, the inner (darker) to the $25 \%-75 \%$ percentiles, the middle line to the median and the whiskers represent the minimum and maximum. On top of each box, the mean value is presented. Finally, in the labels, $\mathrm{OH}$ and $\neg \mathrm{OH}$ stand for with and without the overhearing feature, and RTX $n$ expresses the number of re-transmissions performed.

of unavailable nodes. The traditional choice of retransmitting a packet multiple times (ARQ) generally cannot achieve all three, and therefore more advanced solutions are required. Taking advantage of the support for increased reliability provided by IEEE Std 802.15.4-2015 TSCH we have developed the PAREO methodology to provide end-to-end reliability and high availability over a Track. Through PAREO, we have shown that taking advantage of the shared nature of the wireless medium, and pairing ARQ with multi-path transmissions and promiscuous overhearing allows bridging this gap to a large extent.

As part of our ongoing work in this research direction, the next steps involve the energy efficiency of this solution, especially regarding the overhearing function.

\section{ACKNOWLEDGMENT}

Experiments presented in this paper were carried out using the Grid' 5000 testbed, supported by a scientific interest group hosted by Inria and including CNRS, RENATER and several Universities as well as other organizations (see https://www.grid5000.fr). Additionally, this work was partially performed and supported under the TPI ANR-17-CE10-0007-01 project of the French National Research Agency.

\section{REFERENCES}

[1] A. A. Kumar S., K. Ovsthus, and L. M. Kristensen., "An Industrial Perspective on Wireless Sensor Networks - A Survey of Requirements, Protocols, and Challenges," IEEE Communications Surveys Tutorials, vol. 16, no. 3, pp. 1391-1412, Third 2014.

[2] A. Frotzscher, U. Wetzker, M. Bauer, M. Rentschler, M. Beyer, S. Elspass, and H. Klessig, "Requirements and current solutions of wireless communication in industrial automation," in 2014 IEEE International Conference on Communications Workshops (ICC), June 2014, pp. 67-72.

[3] E. Grossman, "Deterministic Networking Use Cases," RFC 8578, 2019.
[4] "IEEE Standard for Low-Rate Wireless Personal Area Networks (LR-WPANs)," IEEE Std 802.15.4-2015, April 2016.

[5] P. Thubert and G. Papadopoulos, "Reliable and Available Wireless Problem Statement," IETF, I-D draft-pthubert-raw-problem-statement-04, Oct. 2019.

[6] P. Thubert, D. Cavalcanti, X. Vilajosana, and C. Schmitt, "Reliable and Available Wireless Technologies," IETF, I-D draft-thubert-rawtechnologies-03, Jul. 2019.

[7] P. Thubert, "An Architecture for IPv6 over the TSCH mode of IEEE 802.15.4," IETF, I-D draft-ietf-6tisch-architecture-27, 2019.

[8] N. Finn, P. Thubert, B. Varga, and J. Farkas, "Deterministic Networking Architecture," RFC 8655, Oct. 2019.

[9] R. Alexander, A. Brandt, J. Vasseur, J. Hui, K. Pister, P. Thubert, P. Levis, R. Struik, R. Kelsey, and T. Winter, "RPL: IPv6 Routing Protocol for Low-Power and Lossy Networks," RFC 6550, Mar. 2012.

[10] D. Barthel, J. Vasseur, K. Pister, M. Kim, and N. Dejean, "Routing Metrics Used for Path Calculation in Low-Power and Lossy Networks," RFC 6551, Mar. 2012.

[11] G. Fairhurst and L. Wood, "Advice to link designers on link Automatic Repeat reQuest (ARQ)," RFC 3366, August 2002.

[12] G. Z. Papadopoulos, R. Koutsiamanis, N. Montavont, and P. Thubert, "Exploiting Packet Replication and Elimination in Complex Tracks in LLNs," IETF, draft-papadopoulos-raw-pareo-reqs-01, January 2020.

[13] R. A. Koutsiamanis, G. Z. Papadopoulos, X. Fafoutis, J. M. Del Fiore, P. Thubert, and N. Montavont, "From Best-Effort to Deterministic Packet Delivery for Wireless Industrial IoT Networks," IEEE Transactions on Industrial Informatics, vol. 14, pp. 4468 - 4480, 2018.

[14] R. A. Koutsiamanis, G. Z. Papadopoulos, N. Montavont, and P. Thubert, "Common Ancestor Objective Functions and Parent Set DAG Metric Container Extension," IETF, draft-ietf-roll-nsa-extension-04, July 2019.

[15] G.-W. Lee and E.-N. Huh, "Reliable data transfer using overhearing for implicit ack," 2009 ICCAS-SICE. IEEE, p. 19761979, 2009.

[16] Q. Le, T. Ngo-Quynh, and T. Magedanz, "RPL-based multipath Routing Protocols for Internet of Things on Wireless Sensor Networks," In Proc. Int. Conf. on Advanced Technologies for Communications (ATC'14), 2014.

[17] P. Minet, I. Khoufi, and A. Laouiti, "Increasing Reliability of a TSCH Network for the Industry 4.0," In Proc. IEEE NCA, 2017.

[18] Y. Jin, U. Raza, and M. Sooriyabandara, "BOOST: Bringing Opportunistic ROuting and Effortless-Scheduling to TSCH MAC," in 2018 IEEE Global Communications Conference (GLOBECOM), Dec 2018, pp. 1-7. 